\title{
A narrative review of the health disparities associated with malignant pleural mesothelioma
}

\author{
Devon C. Freudenberger, Rachit D. Shah \\ Division of Cardiothoracic Surgery, Virginia Commonwealth University School of Medicine, Richmond, VA, USA \\ Contributions: (I) Conception and design: None; (II) Administrative support: None; (III) Provision of study materials or patients: None; (IV) \\ Collection and assembly of data: None; (V) Data analysis and interpretation: None; (VI) Manuscript writing: Both authors; (VII) Final approval of \\ manuscript: Both authors. \\ Correspondence to: Rachit Shah. Division of Cardiothoracic Surgery, Virginia Commonwealth University School of Medicine, Richmond, VA, USA. \\ Email: rachit.shah@vcuhealth.org.
}

\begin{abstract}
Malignant pleural mesothelioma (MPM) is a cancer of the mesothelial lining of the pleura that has traditionally been associated with asbestos exposure in an industrial setting. Asbestos usage has fortunately been banned or phased out in most industrialized countries resulting in its decline in countries such as the United States. Despite this, MPM continues to place significant burden on its affected patients resulting in overall poor prognosis and survival. Questions arise as to what factors, especially what health disparities, contribute to the disease's dismal prognosis. This article will present a narrative review of recent literature that identifies the impact age, sex, race, access to medical centers, and economics have on the diagnosis, treatment, and prognosis of MPM. As will be discussed, research has shown that factors including younger age, female sex, non-white race, private insurance, Medicare, and higher income have been associated with better survival in MPM. Whereas older age, male sex, white race, lack of insurance, and lower income are associated with worse survival. The identification of these and other health disparities related to MPM may allow for future research, clinical guidelines, and policies to be implemented to decrease the burden health disparities create in the diagnosis, treatment, and prognosis of patients with MPM.
\end{abstract}

Keywords: Malignant pleural mesothelioma (MPM); health disparities

Submitted Dec 14, 2020. Accepted for publication Apr 08, 2021.

doi: $10.21037 /$ jtd-20-3516

View this article at: http://dx.doi.org/10.21037/jtd-20-3516

\section{Introduction}

Mesothelioma is a rare cancer of the mesothelial cells forming the mesothelial lining of multiple organs in the body, including the pleura, peritoneum, pericardium, and tunica vaginalis of the testes. Malignant pleural mesothelioma (MPM) is the most common and accounts for over $80 \%$ of cases (1). Unlike other cancers of the lung, it is not associated with smoking. It is associated with occupational and environmental exposures to asbestos. Traditionally, the industries with the most exposure to asbestos were mining, milling, construction, manufacturing, and ship building. Many industrialized countries have either banned or phased out the use of asbestos over the decades, with many exposures now related to maintenance and remediation of older buildings. However, there are countries that still extensively manufacture and utilize the material, such as China, India, Russia, and Brazil (2).

In the United States the incidence of mesothelioma has been decreasing since the 1970s. From the National Cancer Data Base, there were 19,134 cases of MPM reported from 2004-2013 with an average of 1,800-2,000 new cases reported each year (3). The mortality rate in the United States has also been significantly declining. In a 2018 study analyzing the World Health Organization mortality database from 1994-2013, it was shown that mortality rates among men were significantly declining in the United Kingdom, Lithuania, Sweden, France, the Netherlands, United States, 
Table 1 Search terms used to identify articles

Malignant pleural mesothelioma
Mesothelioma
Incidence
Prognosis
Survival
Mortality
Sex
Gender
Male vs. female
Age
Elderly
Race
Health disparities
Determinants of health
Healthcare
Insurance
Affordable Care Act
Access to healthcare

South Africa, and Australia. Whereas mortality rates among men were significantly rising in Poland, Croatia, Slovenia, Spain, Mexico, Argentina, Hong Kong, Japan, and the Republic of Korea (4). This difference in mortality rates has been related to the timing of asbestos bans in countries. Countries which restricted the use of asbestos in the 1970s, such as the United States, have had down trending mortality rates. In comparison countries that banned asbestos in the 1990s or later, such as Japan, Croatia, Poland, and Hong Kong, have experienced increasing mortality rates (4). This emphasizes that, although MPM remains a rare disease, it remains a disease that patients and the medical community will continue to encounter.

MPM has an overall poor prognosis and survival. The disease has a long latency period of 20-40 years from exposure. Unfortunately, MPM is typically diagnosed at a late stage precluding many individuals from receiving the current recommended treatment modalities. However, subsets of patients are eligible for treatment. Although a thorough discussion of the specifics regarding the diagnosis and management of MPM is beyond the scope of this article, broadly speaking MPM treatment can include a combination of chemotherapy, radiation therapy, and surgery depending upon the stage of the disease. Despite treatment, there is still a dismal prognosis for MPM with median overall survival of 1 year from diagnosis and 5 -year overall survival at approximately $10 \%$ (1). This raises questions as to what factors, specifically health disparities, may be affecting MPM diagnosis, treatment, and prognosis.

This narrative review will provide an overview exploring the question of how patient age, sex, race, socioeconomics, and patient access to care may affect the diagnosis, treatment, and prognosis of MPM. By understanding these factors and their impact on MPM, more research and efforts can be focused on determining how to lessen and mitigate their impact on the care of patients with MPM. We present the following article in accordance with the Narrative Review Reporting Checklist (available at: http:// dx.doi.org/10.21037/jtd-20-3516).

\section{Methods}

In order to conduct this narrative review, a literature review was conducted utilizing the PubMed Database. Key words and phrases were used and combined with one another to identify relevant articles. Search terms included but were not limited to "malignant pleural mesothelioma," "mesothelioma," "health disparities," "age," "sex/gender," "race," "access to healthcare," "determinants of health," etc. A comprehensive list of terms used is included in Table 1. Published research articles between 1990-2019 were considered. The articles were reviewed and relevant articles that addressed the question of this review, specifically how health disparities affect MPM, including clinical practice guidelines, reviews, prospective studies, retrospective studies, and experimental studies were selected for inclusion. Articles from other countries outside of the United States were eligible for inclusion as well given that MPM is a global disease. Only articles from peer-reviewed sources were included. Articles were excluded from review if they were published prior to 1990, were written in a language other than English, and deemed irrelevant to the topic of this review. Additionally, the citation list of each article was inspected to identify other articles of relevance.

A total of 18 articles were included in this narrative review, all of which were peer-reviewed. Thirteen of the eighteen (72\%) articles included data specific to the United States, with the remaining articles pertaining to global data, and data specific to France, Australia, and Italy. A majority 
of the articles, $72 \%$ (13/18), were retrospective studies, while the remainder included clinical guidelines, reviews, prospective studies, and experimental studies.

\section{Discussion}

\section{Disparities in age}

Malignant mesothelioma is typically a disease of older patients, with the median age of diagnosis being approximately 72 years. This has been contributed to the long latency period of the disease. In one study performed by Enewold et al., a random sample of histologically confirmed MPM cases from the year 2011, identified by the National Cancer Institute's Surveillance Epidemiology and End Results program (SEER), showed that approximately $88 \%$ of patients were diagnosed at 60 years or older (5). As MPM is primarily a disease of the elderly, multiple studies have shown that increased age is associated with poorer prognosis and survival in MPM; whereas younger age is associated with better prognosis and survival (3,5-8).

Despite being a disease of old age, elderly patients are less likely to receive therapy for MPM. A 2017 study from the United States revealed that patients aged 66 years and older consistently were more likely to not be treated for their disease, with only $54 \%$ of these individuals receiving systemic therapy (5). Similarly, increasing age has been shown to be a significant factor for low compliance to national guidelines for the treatment of MPM. In a study of 3,419 patients, patients 80 years and older were at the highest rate for low compliance in following the National Comprehensive Cancer Network (NCCN) guidelines with $41 \%$ of these patients not being treated (9).

This raises the question as to why elderly patients with MPM are being sub-optimally treated. It might be inferred that the elderly are less likely to receive any type of treatment given their higher incidence of comorbidities and presumed intolerance to the side effects of treatment. Comorbidities, as defined in the Enewold et al. study by the Charlson comorbidity index, however, were not independently associated with whether or not elderly patients received treatment and were also not independently associated with survival among MPM patients (5).

If comorbidities are not associated with lack of treatment, then other factors must be contributing to this. Clinical nihilism, or the belief that treatment is futile or will cause more harm than good, has been previously suggested. In a survey conducted in Australia distributed to clinicians and nurses involved in the care of MPM patients, clinical nihilism was identified as a perceived barrier to patients receiving chemotherapy. Of the clinicians surveyed, $70 \%$ believed clinician nihilism was a barrier to treatment. This is compared to $29 \%$ of clinicians and $16 \%$ of nurses believing patient nihilism to their diagnosis is a barrier to receiving chemotherapy treatment (10). Perhaps underlying physician bias is a contributing factor as to whether elderly patients are receiving appropriate treatment for MPM.

\section{Disparities in sex}

MPM generally afflicts men more than women due to men historically having more occupational exposure to asbestos. Men were more often employed in the milling, architectural, and ship-building industries than women, leading to higher rates of exposure. Women, however, were traditionally at risk of exposure to asbestos if their spouses worked in the above-mentioned fields and brought home asbestos on their clothing.

In a study analyzing confirmed MPM cases reported from the SEER database from 1973-2009, 78\% of cases were men and 22\% were women, reflecting the historically higher rate of asbestos exposure in men compared to women. Since the early 2000s, the incidence of MPM in men plateaued at 1.8 cases per 100,000 people, and in women 0.4 cases per 100,000 people (11). Another study of similar caliber analyzing the SEER database from 1973-2012 showed that on average, rates of MPM in females were five to six times lower compared to their male counterparts (12).

Although MPM is more prevalent in men than women, female sex has been shown multiple times to be associated with better overall survival $(3,6,8,11)$. In a SEER database study from 2015, Taioli et al. revealed that female sex was independently associated with greater survival than male sex (adjusted HR 0.79, 95\% CI: 0.75-0.82) (6). Interestingly, a study from France showed similar results that women had significantly longer median overall survival of 18.2 months compared to men at 14.1 months $(\mathrm{P}<0.001)$ and when survival was adjusted for age and comorbidities, female sex continued to be a positive prognostic factor for survival (adjusted HR 0.78, 95\% CI: 0.67-0.92, $\mathrm{P}<0.01)(8)$.

It has been suggested that lead-time bias may explain better survival in females due to females seeking medical attention earlier than men. However, when analyzing survival among males and females at 1 and 5 years from diagnosis, females continued to have statistically significant greater survival compared to men stage for stage (11). A 
study performed by Van Gerwen et al. corroborated this finding in an analysis of over 16,000 patients with MPM, where stage at diagnosis was not significantly different between males and females suggesting that lead-time bias is may not be a contributor for the survival differences between males and females that have been observed (13).

Additionally, recent research has been focused on elucidating what genetic factors may be at play for female sex conferring better survival. One such study has looked at the effects of estrogen and estrogen receptor-beta expression on MPM. High estrogen receptor-beta expression on MPM tumors has been shown to have better survival compared to low estrogen receptor-beta expression (14). Circulating estrogen levels vary depending on sex, age, and menopausal status in women. This raises the question as to whether women have better survival than men in MPM because they usually have higher levels of estrogen than men. In their large-scale study, van Gerwen et al. demonstrated that younger women had the highest rates of survival among all groups of male versus female patients with epithelial type histology MPM. This finding is important in supporting the notion that higher levels of circulating estrogen and estrogen receptor-beta expression may confer greater survival. This is an avenue that can be further explored possibly in the setting of potential treatment options.

The receipt of treatment between males and females has also been analyzed. One study analyzing over 3,000 cases of MPM showed that females were $21 \%$ more likely to not receive treatment compared to men; whereas treatment has been shown to be similar between males and females in other studies $(9,11,13)$.

\section{Disparities in race}

As with many other diseases, patient race has been shown in MPM to have differing effects. MPM has higher incidence among white patients than black patients and patients of other races. Approximately $93 \%$ of MPM cases are in white patients, with the incidence of MPM being 1.1 cases per 100,000 white persons and 0.5 cases per 100,000 black persons when analyzing data in the SEER database (15). Additionally, white race has been shown to be independently associated with overall worse survival (adjusted HR 1.24, 95\% CI, 1.18-1.30, $\mathrm{P}<0.001$ ) (3).

Black patients are more likely to be diagnosed at later stages of the disease than white patients $(15,16)$. This may reflect disparities in access to appropriate medical care, lack of insurance amongst this population, or differences in the onset of symptoms in different races which hinder their ability to undergo a timely diagnosis.

Studies have shown that black patients with MPM are also less likely to undergo surgery for MPM compared to whites $(15,16)$. Eighteen percent of blacks received cancer-directed surgery compared to $24 \%$ of whites in data analyzed from the SEER database from 1973 to $2009(\mathrm{P}<0.001)(15)$. In a similar study from 2010, analyzing SEER data from 1990 to 2004 , black patients had a $14 \%$ rate of receiving cancerdirected therapy compared to $23 \%$ of white patients and $21 \%$ of patients of all other races $(\mathrm{P}<0.0001)$. When compared to white patients, blacks had an adjusted OR of receiving surgical treatment of 0.45 (95\% CI: $0.32-0.65$ ) versus 0.77 (95\% CI: $0.53-1.11$ ) for all other races (16). Again this raises questions as to whether this is due to an advanced stage of disease at the time of diagnosis, lack of timely access to medical care, lack of access to large tertiary or academic centers, lack of insurance coverage etc. Further studies should be undertaken to elucidate the factors affecting the diagnosis and treatment of black patients in order to better identify and medically treat these patients.

\section{Disparities in economics}

Health insurance and coverage is a contentious topic in today's political environment, especially in the United States. The articles included in this review that discussed health insurance and coverage were limited to studies from the United States, highlighting how insurance or lack of insurance affects MPM. It goes without surprise that health insurance coverage is an important disparity present in the management of MPM. Uninsured patients are less likely to receive treatment for MPM than patients with private insurance (OR 1.93, 95\% CI: 1.03-3.62, $\mathrm{P}=0.040$ ) or Medicare (OR 2.05, 95\% CI: 1.08-3.86, $\mathrm{P}=0.027$ ) (9). Additionally, it has been shown that patients with Medicaid or no insurance are at higher risks of worse survival than patients with Medicare or private insurance (17). Privately insured patients are more likely to have higher income and to live in more advantaged areas with greater access to medical resources than uninsured and Medicaid patients. Higher patient income has been associated with increased survival $(3,17)$. For example, Saddoughi et al., showed that a median income of $\$ 63,000$ or more was associated with better survival (adjusted HR 0.84, 95\% CI: 0.78-0.90, $\mathrm{P}<0.001$ ) (3).

Since the passage of the Patient Protection and Affordable Care Act in 2010, an estimated 7-16 million 
Table 2 Summary of factors associated with worse and better survival in MPM

\begin{tabular}{ll}
\hline Better survival & Worse survival \\
\hline Younger age & Older age \\
Female sex & Male sex \\
Non-white race & White race \\
Private insurance/Medicare & Medicaid/uninsured \\
Income $>\$ 63,000$ & Income $<\$ 63,000$ \\
\hline
\end{tabular}

MPM, Malignant pleural mesothelioma

individuals gained insurance coverage in the first five years of its implementation (18). As more and more individuals gain insurance coverage, its impact on the diagnosis, treatment, and prognosis of MPM will need to be studied and quantified. Perhaps greater health insurance coverage will be a factor in lessening the impact of the other disparities that currently affect patients with MPM.

\section{Disparities in access to medical centers}

According to guidelines published by the NCCN in the United States, once diagnosed, MPM should be managed by a multidisciplinary medical team composed of medical, radiation, and surgical oncologists with expertise in MPM (1). However, not all patients may have access to medical centers where multidisciplinary teams exist. Twenty-one percent of surveyed physicians in Australia reported lack of access to a specialist center with expertise in MPM as a perceived barrier to patients receiving chemotherapy (10).

Multidisciplinary medical teams and disease specialists are frequently more common to be found at high-volume, academic, or tertiary medical centers. These centers are more likely to have all treatment modalities available for patients including chemotherapy, radiation therapy, and surgery. The highest likelihood of compliance with NCCN guidelines for the treatment of MPM was associated with treatment received at high-volume and academic medical centers as demonstrated in a 2019 study by EspinozaMercado et al. Compared to low-volume centers, highvolume centers had better rates of compliance by a factor of 3.5 (OR 3.58, 95\% CI: 2.34-5.49, $\mathrm{P}<0.001$ ). Academic centers also had a $57 \%$ likelihood of complying to guidelines compared to nonacademic centers (OR 1.57, 95\% CI: $1.30-1.90, \mathrm{P}<0.001)(9)$. These findings suggest that patients without access to these medical centers are being inappropriately undertreated for their disease due to lack of availability to these resources. Lack of equitable access to medical resources and treatment is a problem pervasive in today's society that will only continue to enforce and potentially widen the disparities among patient populations.

Not only is the lack of access to medical centers with multidisciplinary teams a factor affecting diagnosis and treatment of MPM, it is the utilization of these teams by individual physicians. For example, in Australia national guidelines recommend the presentation of all MPM patients at a multidisciplinary meeting, however, physicians reported only $75 \%$ of their personal patients were discussed and less than $50 \%$ of their colleagues' patients were discussed (10). This emphasizes that efforts can be undertaken to ensure that more, and ideally all, patients are discussed at these meetings so that patients can receive the best care possible.

\section{Summary}

Although MPM remains a rare disease, the above narrative review has identified some of the disparities that currently affect the different populations that are afflicted with the disease (Table 2). As MPM continues to be a malignancy that patients and physicians will encounter, it will be imperative to continue to investigate and identify the factors that are contributing to these disparities in order to optimize the overall prognosis and outcomes of patients with MPM. It must be noted that a majority of the articles and data discussed in this review were specific to the United States and thus excludes deeper discussion into the possible health disparities unique to other countries. This emphasizes that further research can be undertaken to identify disparities specific to other populations afflicted with this disease. Identifying these disparities can help guide further research, clinical guidelines, and policies that will allow for the more equitable diagnosis and treatment of this disease.

\section{Acknowledgments}

Funding: None.

\section{Footnote}

Provenance and Peer Review: This article was commissioned 
by the Guest Editors (Virginia Litle and Kei Suzuki) for the series "Socioeconomic Disparities in the Treatment of Thoracic Malignancies" published in Fournal of Thoracic Disease. The article has undergone external peer review.

Reporting Checklist: All authors have completed the Narrative Review Reporting Checklist. Available at: http://dx.doi. org/10.21037/jtd-20-3516

Peer Review File: Available at http://dx.doi.org/10.21037/jtd20-3516

Conflicts of Interest: Both authors have completed the ICMJE uniform disclosure form (available at http://dx.doi. org/10.21037/ jtd-20-3516). The series "Socioeconomic Disparities in the Treatment of Thoracic Malignancies" was commissioned by the editorial office without any funding or sponsorship. The authors have no other conflicts of interest to declare.

Ethical Statement: The authors are accountable for all aspects of the work in ensuring that questions related to the accuracy or integrity of any part of the work are appropriately investigated and resolved.

Open Access Statement: This is an Open Access article distributed in accordance with the Creative Commons Attribution-NonCommercial-NoDerivs 4.0 International License (CC BY-NC-ND 4.0), which permits the noncommercial replication and distribution of the article with the strict proviso that no changes or edits are made and the original work is properly cited (including links to both the formal publication through the relevant DOI and the license). See: https://creativecommons.org/licenses/by-nc-nd/4.0/.

\section{References}

1. National Comprehensive Cancer Network. (2020, October). Malignant Pleural Mesothelioma: NCCN Evidence Blocks (Version 2.2020). Available online: https://www.nccn.org/professionals/physician_gls/pdf/ mpm_blocks.pdf

2. Marsili D, Terracini B, Santana V, et al. Prevention of asbestos-related disease in countries currently using asbestos. Int J Environ Res Public Health 2016;13:494.

3. Saddoughi SA, Abdelsattar ZM, Blackmon SH. National trends in the epidemiology of malignant pleural mesothelioma: A national cancer data base study. Ann
Thorac Surg 2018;105:432-7.

4. Abdel-Rahman O. Global trends in mortality from malignant mesothelioma: analysis of WHO mortality database (1994-2013). Clin Respir J 2018;12:2090-100.

5. Enewold L, Sharon E, Thomas A. Patterns of care and survival among patients with malignant mesothelioma in the United States. Lung Cancer 2017;112:102-8.

6. Taioli E, Wolf AS, Camacho-Rivera M, et al. Determinants of survival in malignant pleural mesothelioma: A surveillance, epidemiology, and end results (SEER) study of 14,228 patients. PLoS One 2015;10:e145039.

7. Wolf AS, Richards WG, Tilleman TR, et al. Characteristics of malignant pleural mesothelioma in women. Ann Thorac Surg 2010;90:949-56; discussion 956.

8. Chouaid C, Assié JB, Andujar P, et al. Determinants of malignant pleural mesothelioma survival and burden of disease in France: a national cohort analysis. Cancer Med 2018;7:1102-9.

9. Espinoza-Mercado F, Borgella JD, Berz D, et al. Disparities in compliance with national guidelines for the treatment of malignant pleural mesothelioma. Ann Thorac Surg 2019;108:889-96.

10. Warby A, Dhillon HM, Kao S, et al. Managing malignant pleural mesothelioma: experience and perceptions of health care professionals caring for people with mesothelioma. Support Care Cancer 2019;27:3509-19.

11. Taioli E, Wolf AS, Camacho-Rivera M, et al. Women with malignant pleural mesothelioma have a threefold better survival rate than men. Ann Thorac Surg 2014;98:1020-4.

12. Glynn ME, Keeton KA, Gaffney SH, et al. Ambient asbestos fiber concentrations and long-term trends in pleural mesothelioma incidence between urban and rural areas in the United States (1973-2012). Risk Anal 2018;38:454-71.

13. Van Gerwen M, Alpert N, Wolf A, et al. Prognostic factors of survival in patients with malignant pleural mesothelioma: an analysis of the National Cancer Database. Carcinogenesis 2019;40:529-36.

14. Pinton G, Brunelli E, Murer B, et al. Estrogen receptor-beta affects the prognosis of human malignant mesothelioma. Cancer Res 2009;69:4598-604.

15. Taioli E, Wolf AS, Moline JM, et al. Frequency of surgery in black patients with malignant Pleural Mesothelioma. Dis Markers 2015;2015:282145.

16. Flores RM, Riedel E, Donington JS, et al. Frequency of use and predictors of cancer-directed surgery in 
the management of malignant pleural mesothelioma in a community-based (Surveillance, Epidemiology, and End Results [SEER]) population. J Thorac Oncol 2010;5:1649-54.

17. Keshava HB, Tang A, Siddiqui HU, et al. Largely unchanged annual incidence and overall survival

Cite this article as: Freudenberger DC, Shah RD. A narrative review of the health disparities associated with malignant pleural mesothelioma. J Thorac Dis 2021;13(6):3809-3815. doi: $10.21037 /$ jtd-20-3516 of pleural mesothelioma in the USA. World J Surg 2019;43:3239-47.

18. French MT, Homer J, Gumus G, et al. Key provisions of the patient protection and Affordable Care Act (ACA): A systematic review and presentation of early research findings. Health Serv Res 2016;51:1735-71. 